\title{
Implementing a policy for pneumococcal prophylaxis in a haematology unit after splenectomy
}

\author{
S G Long, A G Smith, B A Perry, M J Leyland, D W Milligan
}

\begin{abstract}
People who have had a splenectomy for any reason are 40 times more likely to have an overwhelming infection, especially pneumococcal infection, and 17 times more likely to suffer fatal sepsis. The incidence of such life threatening infections is reduced by prophylactic immunisation with polyvalent pneumococcal vaccine and long term antibiotic prophylaxis or instituting prompt antibiotic treatment in the event of fever. This haematology unit agreed a policy of immunisation and antibiotic prophylaxis in June 1988 for all patients undergoing elective splenectomy. The success of this policy was audited in July 1993 by a retrospective analysis of patients' case notes. Seventy four patients were identified as having had a splenectomy, $54(73 \%)$ before June 1988 , of whom only $13(24 \%)$ had received both pneumococcal immunisation and antibiotic prophylaxis before implementation of the agreed policy. At the time of audit, 46/74(62\%) patients were recorded as having received immunisation and 64/74(86\%) as receiving antibiotic prophylaxis or a supply of antibiotics to take in the event of a fever. All but one of the 20 patients who had a splenectomy after June 1988, since implementation of the agreed policy, received immunisation and antibiotic prophylaxis. The authors conclude that establishment of a formal agreed policy for pneumococcal prophylaxis for patients undergoing splenectomy has improved the quality of care.

(Quality in Health Care 1995;4:194-196)
\end{abstract}

Keywords: Prophylaxis, splenectomy, implementing policy.

\section{Introduction}

Splenectomy is a procedure used in haematological practice for both diagnostic and therapeutic purposes. A long term risk for people without a spleen is an increased incidence of overwhelming infection with encapsulated organisms. ${ }^{1}$ From a review of published work Ellison and Fabri concluded that the risk of infection was increased 40-fold and that of fatal sepsis increased 17-fold. ${ }^{2}$
Streptococcus pneumoniae accounts for $70 \%$ of such infections, Haemophilus influenzae and Neisseria meningitidis being responsible for most of the others. ${ }^{1}$

Pneumococcal immunisation and low dose prophylactic penicillin may reduce the incidence of pneumococcal infection in these patients, ${ }^{34}$ and as deaths from pneumococcal septicaemia have been reported many years after splenectomy, prophylactic penicillin should probably be life long. ${ }^{5}$ This is now recommended policy in the United Kingdom for patients who have had a splenectomy. ${ }^{6}$

This unit had no agreed policy on protection against pneumococcal infection for patients who had had a splenectomy. Several infective episodes in patients after splenectomy caused us to review our practice, and in June 1988 a policy was agreed by the consultant staff that all patients undergoing elective splenectomy should receive polyvalent pneumococcal vaccine before the procedure and be offered long term oral phenoxymethyl penicillin (250 mg twice daily). Patients unwilling to take regular long term antibiotics or those allergic to penicillin were given a supply of amoxycillin or erythromycin respectively to take in the event of a fever. We also offered antibiotic prophylaxis to those patients who had already undergone splenectomy and considered immunisation if the previous immunisation status could be ascertained (patients who had been definitely immunised were not reimmunised). From June 1988 medical staff were asked to establish the immunisation status for all patients seen in the clinic who had undergone splenectomy and whether they were taking prophylactic antibiotics. Patients not receiving prophylaxis were given advice about either long term penicillin or immediate antibiotics at the start of any fever, and their general practitioners were informed by letter.

This policy has been sustained by education of new staff and regular discussions at preoutpatient meetings. In July 1993 the success of the implementation of this policy was reviewed.

Patients and methods

The audit review was conducted in July 1993. We searched the following five main sources to identify all patients who had had a splenectomy. 
Histology department-The records of the histology department were searched using SNOP (standardised nomenclature of pathology) codes to identify patients whose spleens had been submitted for histological examination from 1970 to 1993.

Patient Admission System (PAS) - The hospital's diagnostic coding system (based on the Office of Population Censuses and Surveys 4 (OPCS 4)) was searched for patients coded as having been admitted for splenectomy since 1970.

Haematology chemotherapy record charts-All patients undergoing haematological treatment have treatment interventions, including operative interventions such as splenectomy, and response to treatment monitored on standard charts kept separately from the main notes. Those charts from 1984 to 1993 were searched.

Haematology clinics - All doctors were asked to note any patient attending the haematology clinic who had had a splenectomy, and the details were recorded by one of us (SGL).

Ward day book - Records in the ward day book from 1988 to 1993 were searched for all patients who had attended for pneumococcal immunisation.

The case notes of patients identified were then reviewed to confirm splenectomy and to search for documentation of pneumococcal immunisation and antibiotic prophylaxis. General practitioners of patients no longer being followed up were contacted to ascertain whether prophylactic antibiotics were still being taken.

Statistical analysis of the data was performed with the $\chi^{2}$ test with Yates's correction.

\section{Results}

In total, 74 patients were identified as having had a splenectomy, 54 before June 1988 and 20 after that date; 65 continue to be followed up. The table shows the extent of prophylactic immunisation and antibiotic prophylaxis among these patients.

\section{PNEUMOCOCCAL IMMUNISATION}

Of the total patient population at the time of audit $46(62 \%)$ patients were identified as having been immunised with pneumococcal vaccine (Pneumovax I or II) either before or after splenectomy. Of the 54 who underwent splenectomy before June 1988, 13(24\%) were documented as having been immunised before splenectomy and of the 20 who underwent splenectomy after that date $19(95 \%)$ had been immunised, fourteen patients who underwent splenectomy before 1988 but were not immunised at the time were immunised as a result of change in policy in June 1988.

ANTIBIOTIC PROPHYLAXIS

Of the total study population at the time of audit, $64(86 \%)$ patients were recorded as receiving adequate antibiotic cover. In all, $50(68 \%)$ patients take regular prophylaxis, and a further $14(19 \%)$ patients have a supply of antibiotics at home to take if they have a fever. Of the nine patients no longer being followed up, five still take prophylactic antibiotics according to their general practitioners, four were not traced and are assumed for this audit to not be taking antibiotics.

Twenty five $(46 \%)$ patients who had had their splenectomy before June 1988 were recorded as taking prophylactic antibiotics from the time of their operation. All 20 patients who had a splenectomy after June 1988 are taking prophylactic antibiotics. Furthermore, 19 patients who had splenectomy before 1988 and were not receiving adequate antibiotic cover were receiving it in July 1993 at the time of the audit as a result of the change in policy in June 1988.

Overall, patients were 2.5 times more likely to have been immunised with Pneumovax, and 1.45 times more likely to be taking prophylactic antibiotics after introduction of the policy.

\section{NON-UPTAKE OF PROPHYLAXIS}

Twenty eight patients were identified as not having had pneumococcal vaccine or penicillin prophylaxis, or both. No patients were identified who had received pneumococcal vaccine but not penicillin.

\section{Discussion}

Since our formal policy was introduced in June 1988 virtually all patients who subsequently underwent elective splenectomy were given appropriate immunisation and prophylaxis against pneumococcal infection. However, a significant number of patients who had splenectomy before June 1988 have still not been immunised and are not taking prophylactic antibiotics. There are several possible reasons for our failure to extend this policy to this group of patients. Those who had a splenectomy some years ago and are unwilling now to start taking long term daily antibiotic prophylaxis may perceive the risk of infection as low. However, many in this group are willing to have a supply of antibiotics at home to take in the event of a fever. It may be

Prophylactic immunisation and antibiotic cover against pneumococcal infection in patients undergoing elective splenectomy, as ascertained in fuly 1993

\begin{tabular}{lccc}
\hline & $\begin{array}{c}\text { Splenectomy performed } \\
\text { before fune 1988 }\end{array}$ & $\begin{array}{c}\text { Splenectomy performed } \\
\text { after fune 1988 }\end{array}$ & $\begin{array}{c}\text { Total } \\
\text { fun }\end{array}$ \\
\hline No of patients & 54 & 20 & 74 \\
No (\%) receiving immunisation before operation & $13(24)$ & $19(95)$ & $32(43)$ \\
No (\%) receiving immunisation as result of policy & $14(26)$ & 0 & $14(19)$ \\
No (\%) receiving prophylactic penicillin from time of operation & $25(46)$ & 20 & $45(61)$ \\
No (\%) receiving prophylactic penicillin as result of policy & $19(35)$ & 0 & $19(26)$ \\
\hline
\end{tabular}

$\mathrm{p}<0.001$ after June 1988 versus before June 1988 for patients receiving immunisation before operation.

$\mathrm{p}<0.001$ after June 1988 versus before June 1988 for all patients receiving immunisation.

$\mathrm{p}<0.001$ after June 1988 versus before June 1988 for patients receiving prophylactic penicillin from time of operation.

$\mathrm{p}<0.001$ after June 1988 versus before June 1988 for patients receiving prophylactic penicillin as result of policy. 
difficult for medical staff to broach the subject of antibiotic prophylaxis with patients whose splenectomies may have been done several years ago, and in some cases doctors may have simply forgotten to address the issue.

From a retrospective search of case notes it is difficult to be absolutely confident that patients have not received pneumococcal immunisation in the past, as lack of a record cannot be taken as lack of the intervention. When doubt existed, usually because of poor documentation, whether patients had received polyvalent vaccine or not we were reluctant to offer immunisation because of the risk of adverse reactions, including anaphylaxis, in those patients who may have already been immunised. However, we may review this policy because the risk of revaccination is probably much smaller - most reactions are local ${ }^{7}$ - than the risk of death from pneumococcal infection. ${ }^{8}$

The formal policy to ensure that patients are given an effective preventive treatment improved the frequency of the use of a proven effective clinical intervention. We did not study compliance of patients with long term penicillin prophylaxis, and clearly this is another important issue. In view of the serious consequences of pneumococcal infection after splenectomy, the hospital has an obligation to ensure that immunisation is given perioperatively, and all doctors when reviewing these patients, for whatever reason, should assess compliance with antibiotic prophylaxis.

1 Dickerman JD. Splenectomy and sepsis: a warning. Paediatrics 1979;63:938-41.

2 Ellison EC, Fabri PJ. Complications of splenectomy: etiology, prevention and management. Surg Clin North Am 1983;63:1313-30.

3 Zarrabi MH, Rosner F. Rarity of failure of penicillin prophylaxis to prevent post splenectomy sepsis. Arch prophylaxis to prevent post

4 Bolan G, Broome CV, Facklam RR, Plikayatis BD, Fraser DW, Schlech W. Pneumococcal vaccine efficacy in selected populations in the United States. Ann Intern Med 1986;104:1-6.

5 Murdoch IA, Dos Anjos R. Continued need for pneumococcal prophylaxis after splenectomy. Arch Dis Child 1990;65:1268-9.

6 McMullin M, Johnston G. Long term management of patients after splenectomy. $B M F$ 1993;307:1372-3.

7 Grabenstein JD, DICP, Annals of Pharmacotherapy 1989;23:258-9.

8 Kafidi KT, Rotschafer JC. Bacterial vaccines for splenectomized patients. Drug Intelligence and Clinical Pharmacy 198;22:192-7. 